\title{
Mechanical modelling of interfacial phase in coatings
}

Ri Xia ${ }^{1}$, Mingchen $\mathrm{Huo}^{1}$ and Jinquan $\mathrm{Xu}^{1, *}$,

* Correspondence: jqxu@sjtu.edu.cn, Tel.: +86-21-6447-3073

\begin{abstract}
Interface between matrix/coating or coating/coating in fact represents a very complicate thin interfacial layer. So interface model is necessary to avoid the difficulty on considering such a complicate thin layer in analysis. Classic interface model and cohesive model have been widely used in stress analysis of coating materials, though they cannot represent the effect of very thin interfacial layer accurately. A complete interface model has been deduced from the equivalent constitutive of interfacial layer in this paper. It is found that both classic interface model and cohesive model sometimes cannot give correct analysis results, while the complete interface model can always give a correct result. Moreover, the stress parallel to interface within the interfacial layer can also be analyzed by the new model. Besides, this model can also be used to describe the equivalent properties of interfacial layer, thereby, can provide a quantitative characterizing method for interfacial layer itself.
\end{abstract}

Keywords: Interfacial phase; Interface model; Displacement jump; Boundary element method

\section{Introduction}

It is nearly impossible to consider the thin interfacial layer/phase in detail due to its complexity of compositions, as shown in Fig.1, and such an interfacial phase is unavoidable at coatings interface. It is well known that strength behavior of bonded materials is dominated by the interface. The socalled interface behavior/property corresponds to this interfacial layer. Therefore, the interface model must be introduced in analysis [1-3] to take the effect of interfacial layer into account. Till to now, there are two main models to express the effect of interfacial layer in mechanical analysis. The first is the classic interface model [4-6], as shown in Fig.2a. By which the interface layer is regarded as a theoretical plane (without thickness), and continuous conditions of traction and displacement are applied to the interface directly. Almost all theoretical analysis [7-9] of interfacial problems are based on this model. This model comes just from an imaged plane in a continuum, the effect of interfacial layer is expected to be involved in interface strength/toughness. The other is the cohesive model [1012], as shown in Fig.2b. By which the interfacial layer is regarded as springs connecting two materials, with thickness/length of zero. In the cohesive model, there are displacement jumps across the interface, and the traction is considered as being proportional to the jump. A large amount of numerical study [13-15] on interfacial problem based on cohesive model can be found in literature. Both deformation and strength behaviors of interfacial layer are expected to be involved by model constants in the model. However, springs are strictly different from continuum, so the cohesive model contains some intrinsic conflicts with thin continuum layer, as it will be discussed in detail later.

Modelling the interfacial layer as a plane without thickness would be a simple and practical way to deal with it in application, the problem is how to give the mechanical condition at the modeled interface. Regarding the interfacial layer as a thin continuum layer, it is possible to derive the mechanical condition theoretically on interface, from the constitutive of interfacial layer. Furthermore, the deformation of interfacial layer can be considered as within elastic range in engineering analysis. Thereby, the complicated interfacial layer can be considered as an equivalent elastic material to approximate the gradient variation of material within the interfacial layer. However, no matter it is 
46

47

48

49

assumed as elastic or non-elastic, if its equivalent constitutive is known, the mechanical condition can be derived in a similar way, as it will be shown in next section.

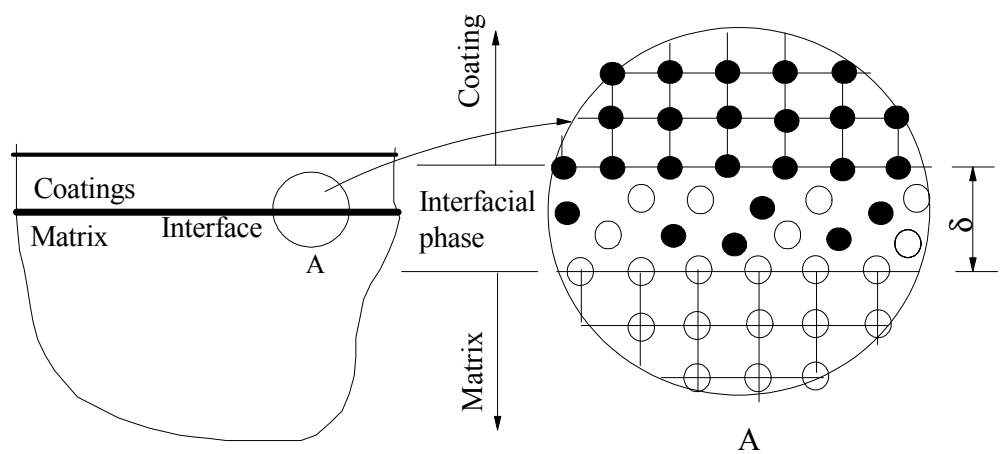

Fig.1 A schematics of interfacial layer

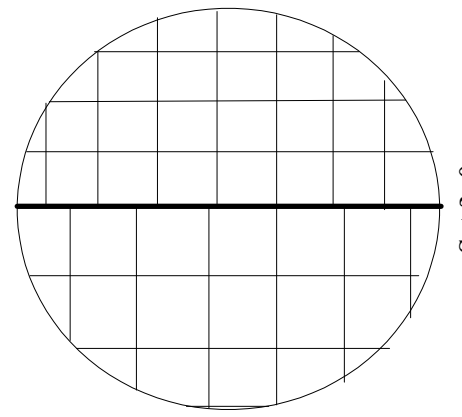

a) Classic interface model

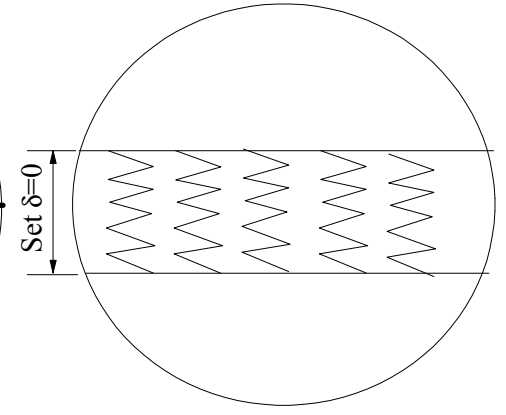

b) Cohesive model

Fig.2 Traditional modelling of interfacial layer

\section{Physical model and interface model}

Considering materials "1", "2" bonded with an intermediate layer, as shown in Fig.3a. Interfaces 1 and 2 are now just the imaged planes in continuum without any interfacial layer, so classic interface model can be applied to them. If the thin intermediate layer (with thickness $\delta$ ) is considered directly, it is just the physical model, and no so-called interface model is necessary. But it would be very inconvenient due to the very thin thickness and gradient material properties of intermediate layer. So it is better to consider all the intermediate layer as a plane(interface) as shown in Fig.3b. Such an interface has left and right sides corresponding interface "1", "2" shown in Fig.3a, respectively. We call it modeled interface below. Not only the displacement, but also the traction, would have jumps across the interface in such an interface model, since the modeled interface corresponds to the intermediate layer in fact.

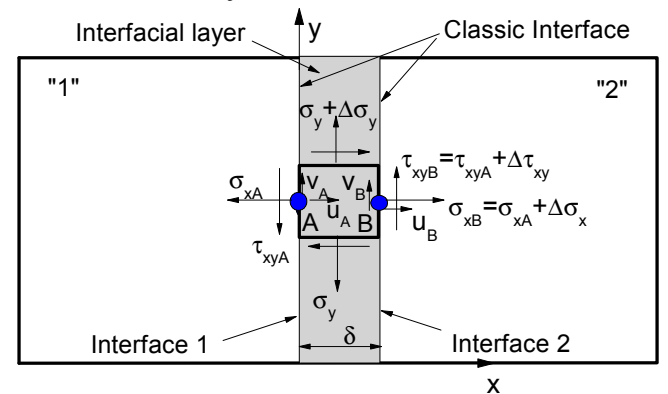

(a) Physical model of bonding with interfacial layer

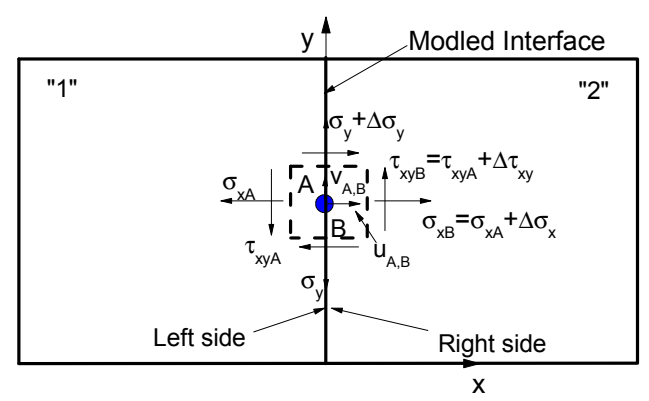

(b) Modeled Interface for analysis

Fig.3 Modelling process of interface

Let $\mathrm{A}$ and $\mathrm{B}$ be the corresponding pair of points at two sides of interfacial layer, they will overlap into a same point at modeled interface, as shown in Fig.3b. Displacements and traction at A and B, i.e., $u_{A}, v_{A}, \sigma_{x A}, \tau_{x y A}$ and $u_{B}, v_{B}, \sigma_{x B}, \tau_{x y B}$, as shown in Fig.3a, will then also refer to a same point at modeled interface. So there are eight interfacial variables in total, $u_{A}, v_{A}, \sigma_{x A}, \tau_{x y A}$ and $u_{B}, v_{B}, \sigma_{x B}, \tau_{x y B}$, at a point of modeled interface in fact. The stress component parallel to interface 
within interfacial layer $\left(\sigma_{y}\right)$ disappears at modeled interface, but it can be revivified if displacement and traction's jumps are allowed at modeled interface. According to the knowledge of continuum mechanics, however, only four unknowns at interface can be solved for plane problems. Thereby, four additional relationships among variables $u_{A}, v_{A}, \sigma_{x A}, \tau_{x y A}$ and $u_{B}, v_{B}, \sigma_{x B}, \tau_{x y B}$ must be prepared as the mechanical conditions at modeled interface.

Denoting

$$
u_{B}=u_{A}+\Delta u, v_{B}=v_{A}+\Delta v
$$

and regarding $u_{A}, \Delta u, v_{A}, \Delta v$ as unknowns to be solved (here $\Delta u$ and $\Delta v$ are displacement jumps at the modeled interface), an interface model must provide following four relationships

$$
\begin{array}{ll}
\sigma_{x A}=f_{1}\left(u_{A}, \Delta u, v_{A}, \Delta v\right), & \tau_{x y A}=f_{2}\left(u_{A}, \Delta u, v_{A}, \Delta v\right) \\
\sigma_{x B}=f_{3}\left(u_{A}, \Delta u, v_{A}, \Delta v\right), & \tau_{x y B}=f_{4}\left(u_{A}, \Delta u, v_{A}, \Delta v\right)
\end{array}
$$

It can be easily found that above relationships are in fact similar to the constitutive of interfacial layer. Thereby, mechanical conditions at modeled interface must be dependent only on the material property of physical interfacial layer. Traditionally, Eq. (2) is usually given directly by assumptions and approximations. For example, in the classical interface model, mechanical conditions at modeled interface are assumed as

$$
\Delta u=\Delta v=0, \quad \sigma_{x A}=\sigma_{x B}, \quad \tau_{x y A}=\tau_{x y B}
$$

No jump of displacement or traction is permitted. It should be noted that these relationships are strict if there is no interfacial layer, i.e., for an imaged plane in continuum. In other words, the classic interface model does not contain the effect of interfacial layer.

In a cohesive model, mechanical conditions are assumed as

$$
\sigma_{x A}=\sigma_{x B}=f_{1}(\Delta u), \quad \tau_{x y A}=\tau_{x y B}=f_{2}(\Delta v)
$$

functions $f_{1}, f_{2}$ can be linear or non-linear here. No jump of traction is permitted. Obviously, Eq. (3a) or (3b) are assumptions or approximations only if they are applied to modeled interface corresponding to an interfacial layer.

To avoid improper assumptions or losing message of physical interfacial layer, it is better to deduce Eq. (2) from the constitutive of interfacial layer theoretically. It is true that the constitutive of thin interfacial layer may be a gradient one, and may be very difficult to be determined. But an equivalent elastic material can be used to approximate it. Once the constitutive has been determined by test/inverse analysis or other methods, mechanical conditions at modeled interface must be dependent on it only. In other words, the interface property can be described by the equivalent elastic material.

\section{The complete interface model}

For the simplicity, an equivalent linear elastic interfacial layer is considered here. The constitutive of interfacial layer can then be expressed as

$$
\begin{gathered}
\sigma_{x}=\frac{\mu}{\kappa-1}\left[(\kappa+1) \frac{\partial u}{\partial x}+(3-\kappa) \frac{\partial v}{\partial y}\right], \tau_{x y}=\mu\left[\frac{\partial u}{\partial y}+\frac{\partial v}{\partial x}\right] \\
\sigma_{y}=\frac{\mu}{\kappa-1}\left[(\kappa+1) \frac{\partial v}{\partial y}+(3-\kappa) \frac{\partial u}{\partial x}\right]
\end{gathered}
$$

where $\mu=E /[2(1+v)]$ is the equivalent shear modulus of interfacial layer ( $E$ : Young's modulus), $v$ is the Poisson's ratio, and $\kappa$ is an elastic constant defined by 
112

114

If the thickness of interfacial layer is very small, one has

$$
\left.\frac{\partial u}{\partial x}\right|_{x=0}=\left.\frac{\partial u}{\partial x}\right|_{x=\delta} \approx \frac{\Delta u}{\delta},\left.\frac{\partial v}{\partial x}\right|_{x=0}=\left.\frac{\partial v}{\partial x}\right|_{x=\delta} \approx \frac{\Delta v}{\delta}
$$

where $\delta$ is the thickness of interfacial layer. Applying Eq. (4) to points A,B, one gets

$$
\begin{gathered}
\sigma_{x A}=\frac{\mu(\kappa+1)}{(\kappa-1) \delta} \Delta u+\left.\frac{\mu(3-\kappa)}{\kappa-1} \frac{\partial v}{\partial y}\right|_{x=0}, \quad \tau_{x y A}=\frac{\mu}{\delta} \Delta v+\left.\mu \frac{\partial u}{\partial y}\right|_{x=0} \\
\sigma_{x B}=\frac{\mu(\kappa+1)}{(\kappa-1) \delta} \Delta u+\left.\frac{\mu(3-\kappa)}{\kappa-1} \frac{\partial v}{\partial y}\right|_{x=\delta}, \quad \tau_{x y B}=\frac{\mu}{\delta} \Delta v+\left.\mu \frac{\partial u}{\partial y}\right|_{x=\delta}
\end{gathered}
$$

Eq. (7) can be degraded into the linear cohesive model if variations of displacement $(\partial u / \partial y, \partial v / \partial y)$ along interface are small enough, but between the spring constants of debonding and shear stress, there should be an intrinsic relationship as indicated in Eq. (7). Obviously, the variations of displacement along interface cannot always be neglected. Taking spring constants arbitrarily and neglecting the variations of displacement along interface would lead to conflicts of cohesive interface model with physical interfacial layer.

On the other hand, Eq. (7) can be used directly as the mechanical condition at the modeled interface. We call the interface model with mechanical conditions shown in Eq. (7) as complete interface model, since it is constitutive-based and no conflict is involved.

By this complete model, the stress component parallel to the interface in the interfacial layer can be expressed as

$$
\sigma_{y}=\frac{\mu(\kappa+1)}{\kappa-1} \frac{1}{2}\left[\left.\frac{\partial v}{\partial y}\right|_{x=0}+\left.\frac{\partial v}{\partial y}\right|_{x=\delta}\right]+\frac{\mu(3-\kappa)}{(\kappa-1) \delta} \Delta u
$$

where following approximation has been adopted

$$
\frac{\partial v}{\partial y}=\frac{1}{2}\left[\left.\frac{\partial v}{\partial y}\right|_{x=0}+\left.\frac{\partial v}{\partial y}\right|_{x=\delta}\right]
$$

If the difference of partial derivatives is large, an interpolation can also be considered instead of Eq. (9) to improve the computation accuracy of $\sigma_{y}$. From above equations, remaining displacements and their partial derivatives at interface 1(i.e, left side of modeled interface), mechanical conditions at modeled interface can be obtained

$$
\begin{aligned}
& \sigma_{x A}=k_{1} \Delta u+k_{12} \frac{\partial v}{\partial y}, \quad \tau_{x y A}=k_{2} \Delta v+k_{21} \frac{\partial u}{\partial y} \\
& \sigma_{x B}=k_{1} \Delta u+k_{12} \frac{\partial v}{\partial y}+k_{12} \frac{\partial \Delta v}{\partial y}=\sigma_{x A}+k_{12} \frac{\partial \Delta v}{\partial y} \\
& \tau_{x y B}=k_{2} \Delta v+k_{21} \frac{\partial u}{\partial y}+k_{21} \frac{\partial \Delta u}{\partial y}=\tau_{x y A}+k_{21} \frac{\partial \Delta u}{\partial y}
\end{aligned}
$$

where constants included in the model are

$$
k_{1}=\frac{\mu(\kappa+1)}{(\kappa-1) \delta}, \quad k_{12}=\frac{\mu(3-\kappa)}{\kappa-1}, \quad k_{2}=\frac{\mu}{\delta}, \quad k_{21}=\mu
$$

So interface model constants reflected both the constitutive and thickness of interfacial layer. That is, to describe the deformation property of interfacial layer, above four constants can be used as the interface constants, but among them only three are independent. From Eqs (10)(11), it can be seen that interface constants are different if the thicknesses of interfacial layer are different, even for the case that the bonded pair is the same. Based on Eq. (11), one can further deal with an interfacial layer with non-uniform thickness just by adjusting model constants $k_{1}$ and $k_{2}$ according to the 
thickness. From Eq. (10), it can be seen that traction's jump is also possible at the modeled interface, if the variation of displacement jump along interface is not small enough.

Partial derivatives of displacements and their jumps appeared in Eq. (10) can be expressed by displacements and their jumps through numerical technology, so Eq. (10) can give the complete conditions for analysis of bonded materials.

It is interesting that Eq. (10) has a form of

$$
\sigma_{x A}=f_{1}(\Delta u, v), \quad \tau_{x y A}=f_{2}(\Delta v, u)
$$

$$
\sigma_{x B}=f_{1}(\Delta u, v)+f_{3}(\Delta v), \quad \tau_{x y B}=f_{2}(\Delta v, u)+f_{4}(\Delta u)
$$

Interactions can be found in these relationships, i.e., normal displacement and its jump at interface can also induce shear stress, and tangential deformation and its jump can also induce normal stress. Such an interaction cannot be dealt with by the cohesive model. These interactions are strongly dependent on property constants of constitutive. For a non-linear interfacial layer, they may become much stronger.

Though above mechanical condition at modeled interface is developed for plane problems, obviously, it is not difficult to extend it to 3 dimensional problems.

\section{Numerical Examinations}

Eq. (10) can be easily coded[16] into a FEM or BEM program. In this study, we have coded it into BEM2D [17] which was developed by authors previously, and corresponding analysis has been carried out. The physical bonding model is shown in Fig.4a, with size of $W=20 \mathrm{~mm}, \delta=0.1 \mathrm{~mm}$. For the purpose of comparison and verification, four analysis models have been considered. One is the model with element divisions for interfacial layer too, as shown in Fig.4b, and the corresponding results will be denoted by 'strict' for convenience. The others are models with the use of classic, complete interface models and the cohesive model. The thickness of interfacial layer has been modeled as zero in later three models. Excluding meshes for interfacial layer, element divisions for classic and complete interface models are the same. The corresponding results will be denoted by 'classic interface mode' and 'modeled interface', respectively. Material constants are shown in Table 1, where constants of complete interface model were calculated from Eq. (11). Two kinds of interfacial layers, hard and soft ones, have been calculated. And two types of loadings, i.e., remote tensile and shear loadings, as shown in Fig. $4 \mathrm{~b}$, have been analyzed. It is noted that constants of cohesive model are determined by matching its analysis results with that obtained by other methods. It is not easy to determine cohesive constants without the reference to other results. So the comparison among different methods will be arranged for 'strict', 'classic' and 'modeled interface' at first. And then the results obtained by cohesive model will be discussed.

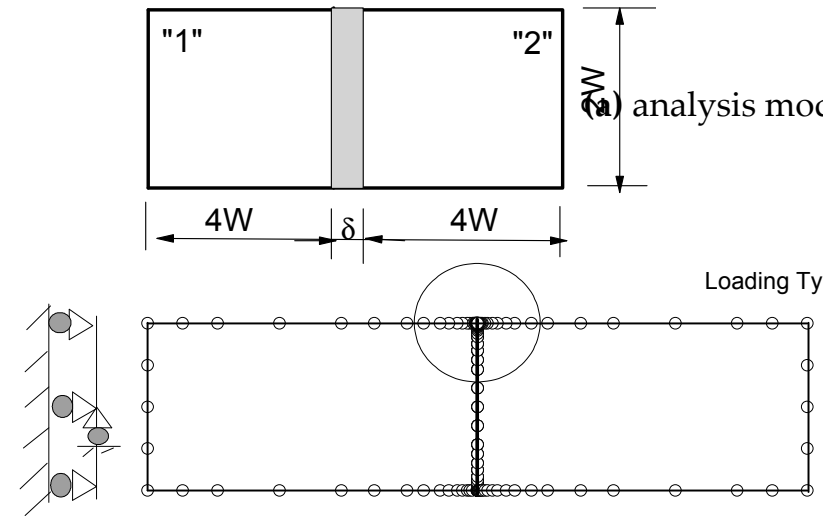

(b) element divisions

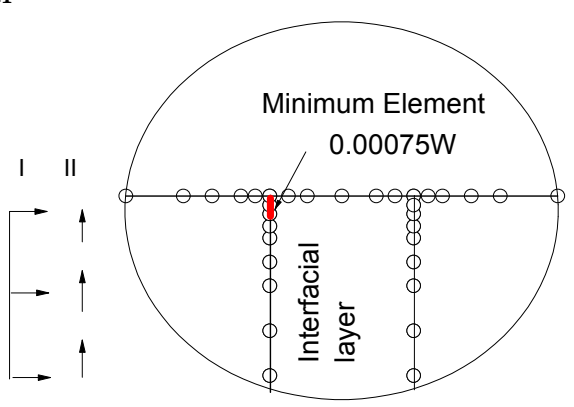

(c) element divisions near the edge

Fig. 4 BEM analysis model and element divisions

Table 1 Material and interface constants 


\begin{tabular}{|c|c|c|c|c|c|c|}
\hline & $E / G P a$ & $v$ & $k_{1}$ & $k_{2}$ & $k_{12}$ & $k_{21}$ \\
\hline & & & \multicolumn{2}{|c|}{$\mathrm{GPa} / \mathrm{mm}$} & \multicolumn{2}{|c|}{ GPa } \\
\hline "1" & 210 & 0.3 & & & & \\
\hline “2” & 304 & 0.27 & & & & \\
\hline Hard Int. Layer & 108 & 0.35 & 1733 & 400 & 93.3 & 40 \\
\hline Soft Int. Layer & 2.9 & 0.38 & 54.3 & 10.51 & 3.33 & 1.051 \\
\hline \multirow[t]{2}{*}{ Cohesive model } & $\begin{array}{l}\text { Hard Int. } \\
\text { Layer }\end{array}$ & \multicolumn{3}{|c|}{$k_{1 \mathrm{co}}=1528 \mathrm{GPa} / \mathrm{mm}$} & $100 G$ & \\
\hline & Soft Int. Layer & \multicolumn{3}{|c|}{$k_{1 c o}=54.3 \mathrm{GPa} / \mathrm{mm}$, } & $.51 G$ & $m m$ \\
\hline
\end{tabular}

\section{5}

Fig.5 shows examples of deformation under remote tensile and shear loadings for a hard interfacial layer obtained by the 'strict' analysis model. The deformations obtained by 'classic' and 'modeled interface' are almost the same as shown in left graphs of Fig.5. From the right graphs of Fig.5, it can be found that deformations of interfacial layer at thickness direction are uniform approximately under both remote tensile and shear loadings. This fact means that the partial derivative $\partial \Delta u / \partial y$ is very small, so the jump of shear stress must also be very small for these examples. While the deformation obtained by a cohesive model is similar only if spring constants are selected as shown in Table 1, otherwise, significant difference might appear.
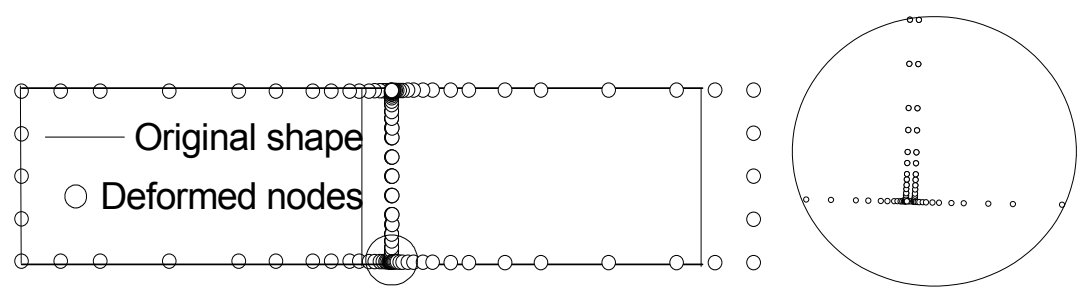

(a) Under tensile loading
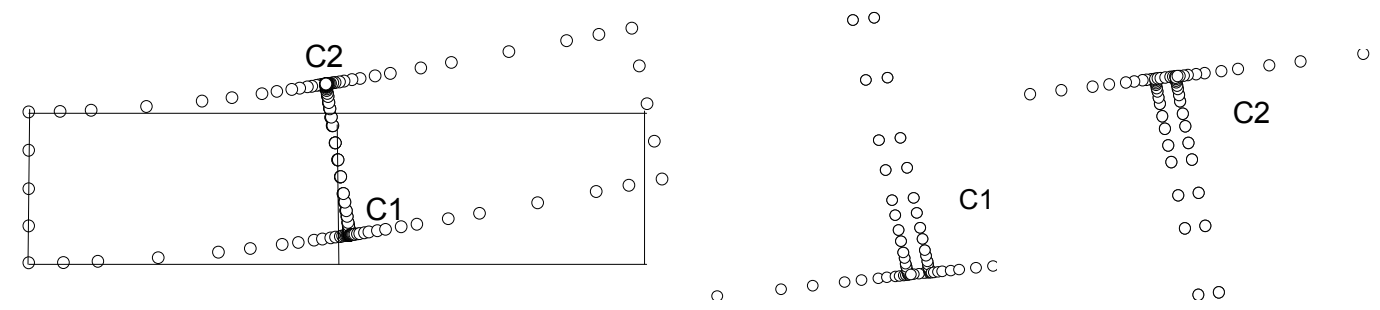

(b) Under shear loading

Fig. 5 Deformations under different loadings with a hard interfacial layer

Fig.6 shows interfacial stresses obtained by different analysis models for the hard interfacial layer. It can be found that results obtained by different analysis models agree well with each other. This fact means that the complete interface model has been correctly coded, and the model can really take the true effect of interfacial layer into account though its thickness has been modeled as zero. It can also be found that the jump of traction at two sides of interfacial layer is generally small, but it increases near to the interface edge. The results obtained by complete interface model agree well with that obtained by 'strict' analysis. It is very interesting that tractions obtained by classic interface model also agree well with that by 'strict' and complete model. This fact means that classic interface model is also accurate enough for a hard interfacial layer if only tractions on interface are concerned. However, if the stress component parallel to interface within the interfacial layer is also concerned, the complete interface model or 'strict' analysis is necessary. Analysis based on classic interface model 
211 cannot give such a result. Moreover, though there is no obvious difference on traction between classic 212 and complete interface models, stresses within matrix or coating, especially the components parallel 213 to interface, might have obvious difference.

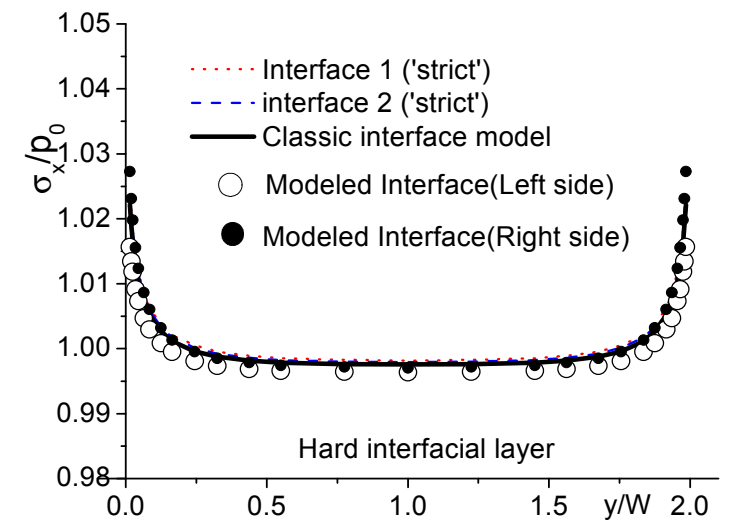

(a) Normal stresses under remote tensile loading

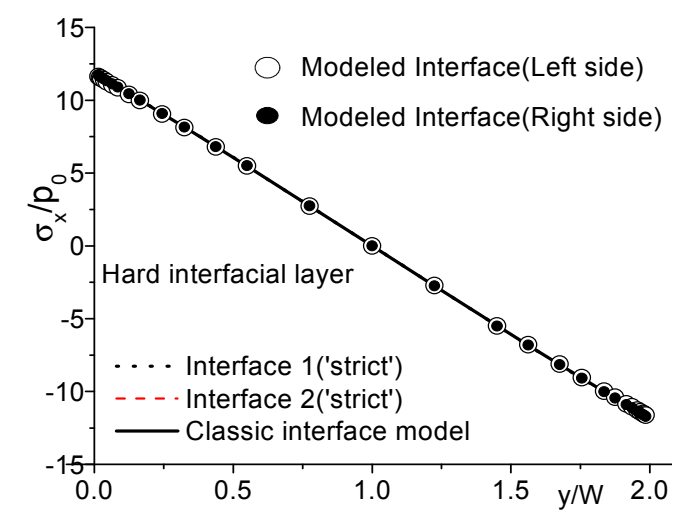

(c) Normal stresses under remote shear loading

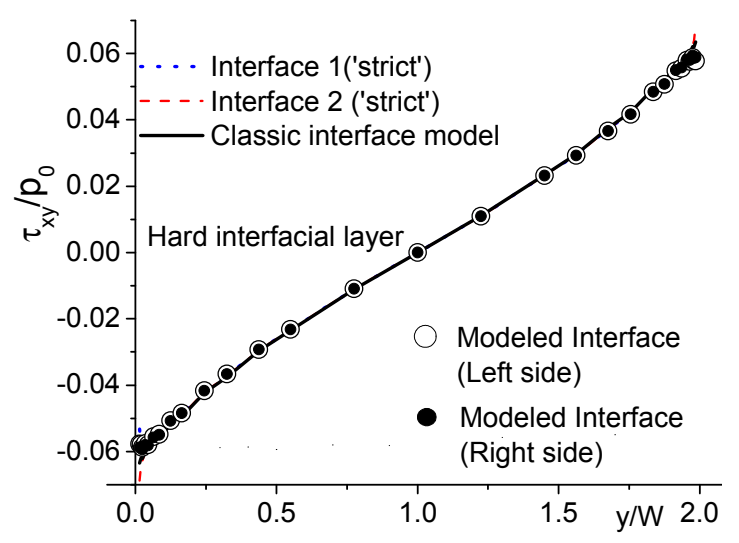

(b) Shear stresses under remote tensile loading

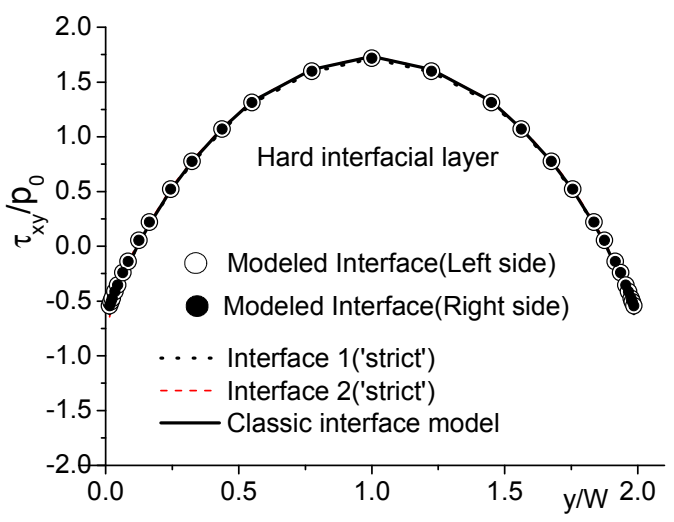

(d) Shear stresses under remote shear loading

Fig. 6 Comparison of interfacial stresses obtained by different analysis types for a hard layer

Fig. 7 shows interfacial stresses obtained by different analysis models for the soft interfacial layer. Unlike the case of hard interfacial layer, though the debonding stresses are almost the same, there is obvious difference between classic interface model and strict analysis. While all results obtained by the complete interface model agree well with that by 'strict' analysis. Therefore, an important conclusion can be obtained. That is, if the interfacial layer is much softer than matrix and coatings, it cannot be regarded as continuous plane, a complete interface model is necessary. The reason is simple, a soft layer can generate large deformation and decrease the constriction on interface between matrix and coating. Moreover, under remote tensile loading, as shown in Fig.7a, classic interface model would lead to stronger edge-singularity of debonding stress than that by 'strict' or complete interface model, while the later two agree with each other. However, under remote shear loading, it seems that classic interface model can also give correct debonding stress. 

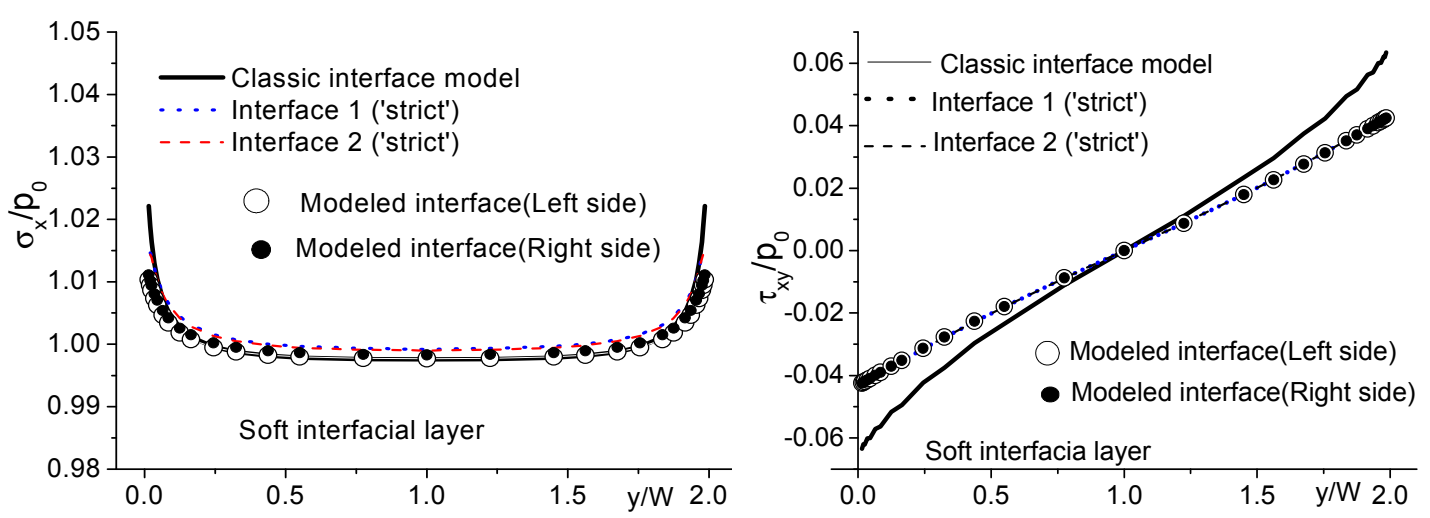

(a) Normal stresses under remote tensile loading

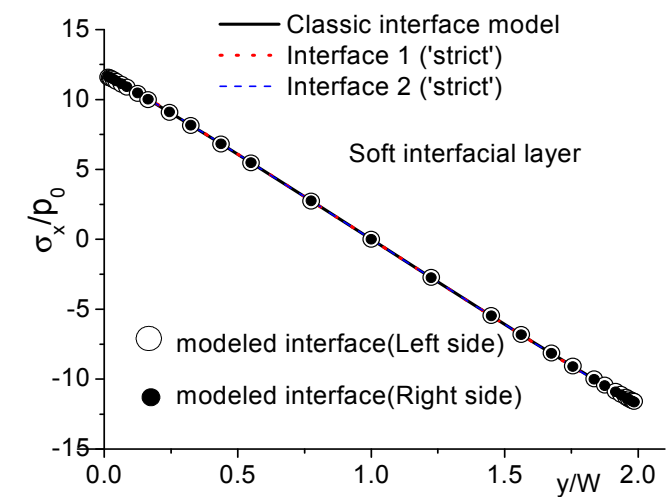

(b) Shear stresses under remote tensile loading

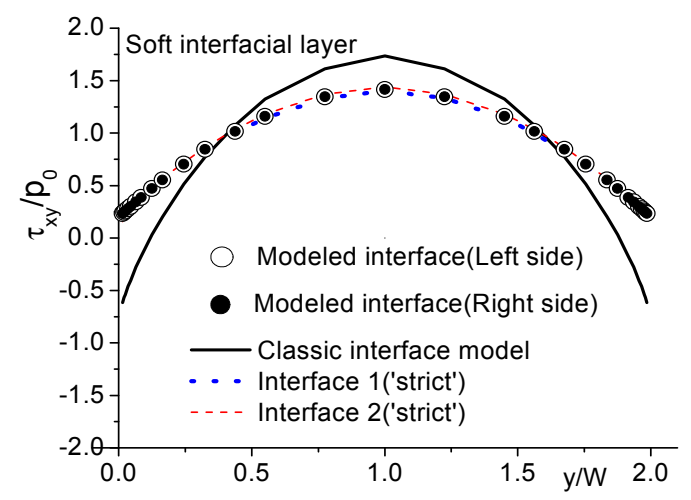

(c) Normal stresses under remote shear loading

(d) Shear stresses under remote shear loading

Fig. 7 Comparison of interfacial stresses obtained by different analysis models for a soft layer

From the results obtained by 'strict' analysis or complete interface model, the relationship between traction and displacement jump can be obtained. The slopes of this relationship correspond to the spring constants of cohesive model. Cohesive model constants shown in Table 1 are obtained in this way. Focusing on traction only, cohesive model can also give reasonable results, as shown in Fig.8. However, it must be noted that the agreement appears only if spring constants are selected just as that shown in Table 1, otherwise, no agreement would appear. It is very interesting that spring constants of cohesive model are the same with $k_{1}, k_{2}$ in complete interface model for a soft interfacial layer, as shown in Table 1. This fact means that the second term in Eq. (10) can be neglected. However, for a hard interfacial layer, though the shear spring constant is still the same with $k_{2}$, the tensile spring constant is different from $k_{1}$. This fact means that the second term in Eq. (10) of debonding stress cannot be neglected, and cohesive model approximated it by $\Delta k_{1} \Delta u$. Anyway, the effect of second term in Eq. (10) may be dependent on stress state, so it is difficult to determine spring constants in cohesive model in a generalized way.

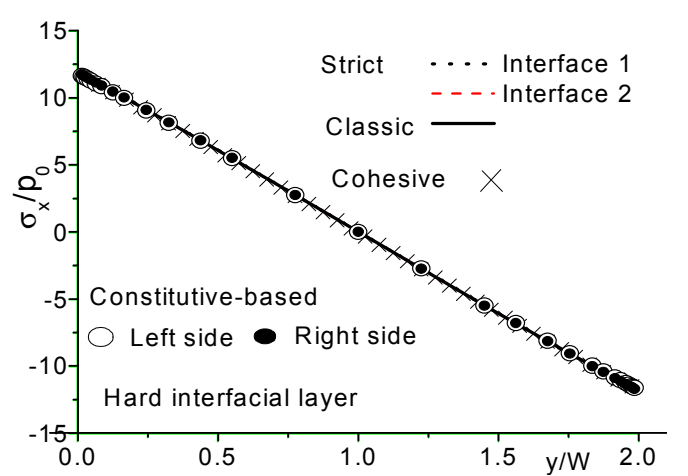

(a) Normal stresses under remote shear loading

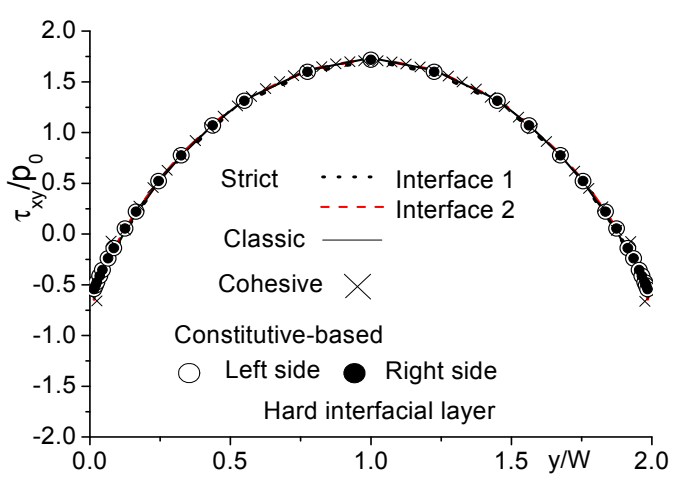

(b) Shear stresses under remote shear loading 


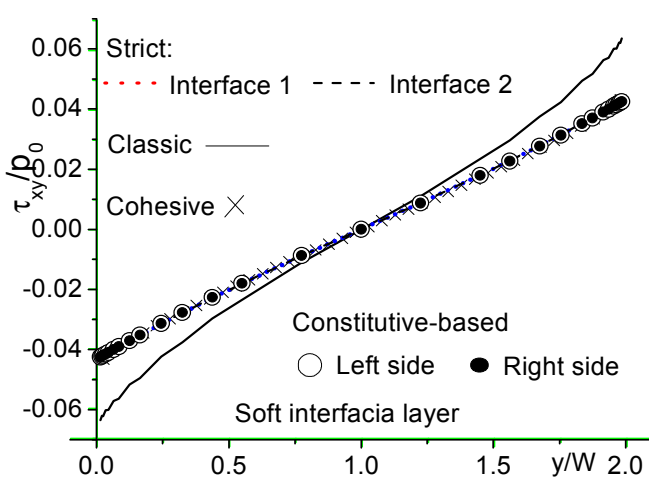

(d) Shear stresses under remote tensile loading

(c) Normal stresses under remote tensile loading

Fig.8 Comparison of interfacial stresses for cohesive and other models

One important advantage of complete interface model is that the stress component parallel to interface can also be analyzed. Fig. 9 shows the stress distribution. The 'strict' result is obtained by BEM analysis for internal points at $x=0.05 \mathrm{~mm}$ in the region of interfacial layer, while the result for complete interface model is calculated from Eq. (8). It can be found that the stress obtained by complete interface model agrees very well with that by 'strict' analysis, no matter the interfacial layer is hard or soft, and no matter the remote loading is tensile or shear. This fact means that the complete interface model has reflected the true effect of interfacial layer completely. It can also be found that a soft interfacial layer would lead to much larger $\sigma_{y}$ than a hard one, and its value is in the same order of normal stress. This fact indicated that the stress parallel to interface within a soft interfacial layer must be taken into consideration for interfacial failure.

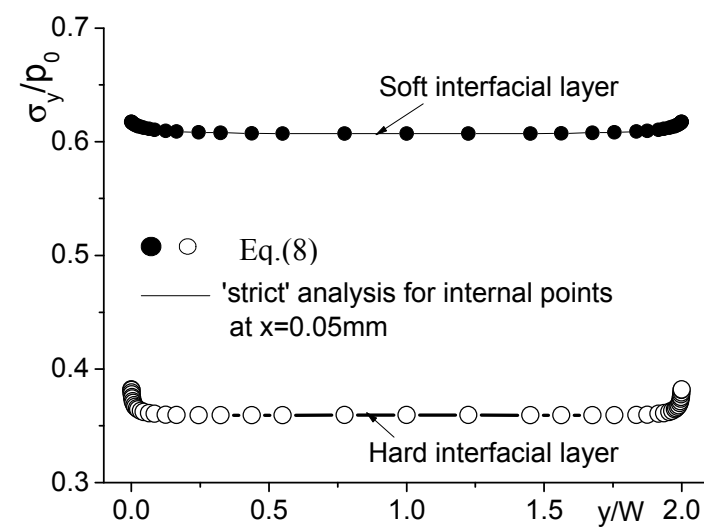

(a) Under remote normal loading

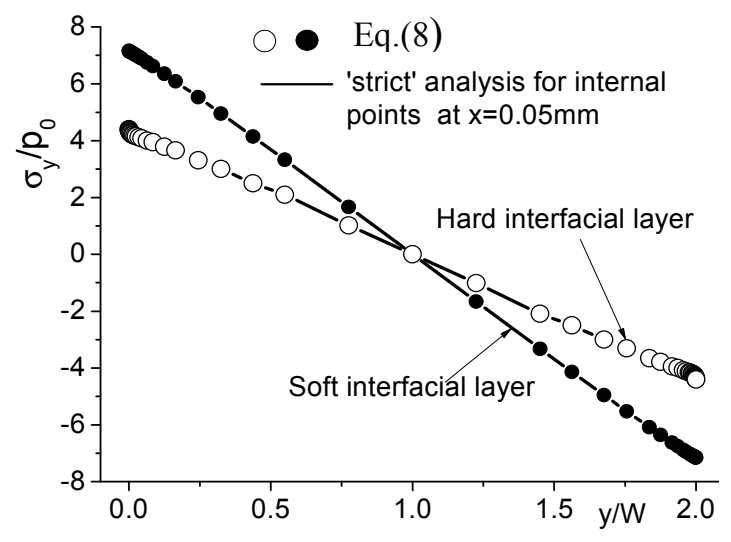

(b) under remote shear loading

Fig. 9 Stresses in interfacial layer parallel to the interface

\section{Discussion}

\subsection{Non-linear interface model}

Non-linear material may be an alternative choice in considering equivalent material of interfacial layer. The interfacial layer can also be modeled as a plane, but the mechanical conditions at modeled interface are very different from Eq.(10).

Assuming that the non-linear constitutive of interfacial layer can be expressed in a power-law way as

$$
\bar{\varepsilon}=\beta \bar{\sigma}^{m}
$$

where $\beta, m$ are material constants, and $\bar{\sigma}, \bar{\varepsilon}$ denote equivalent stress and strain, respectively. Then one can obtain [18] 


$$
\bar{\sigma}^{m-1} \sigma_{x}=\frac{2}{3 \beta}\left(\frac{\partial u}{\partial x}-\frac{\partial v}{\partial y}\right), \quad \bar{\sigma}^{m-1} \sigma_{y}=-\frac{2}{3 \beta}\left(\frac{\partial u}{\partial x}-\frac{\partial v}{\partial y}\right), \quad \bar{\sigma}^{m-1} \tau_{x y}=\frac{1}{3 \beta}\left(\frac{\partial u}{\partial y}+\frac{\partial v}{\partial x}\right)
$$

279

where

where the equivalent stress can be expressed as

$$
\begin{aligned}
& \bar{\sigma}=\sqrt{3} \sqrt{\left(\frac{\sigma_{x}-\sigma_{y}}{2}\right)^{2}+\tau_{x y}^{2}} \quad \text { for plane strain } \\
& \bar{\sigma}=\sqrt{\sigma_{x}^{2}+\sigma_{y}^{2}-\sigma_{x} \sigma_{y}+3 \tau_{x y}^{2}} \quad \text { for plane stress }
\end{aligned}
$$

Substituting Eq. (14) into (15) yields

$$
\bar{\sigma}^{m}=\frac{\sqrt{3}}{3 \beta} \sqrt{4\left(\frac{\partial u}{\partial x}-\frac{\partial v}{\partial y}\right)^{2}+\left(\frac{\partial u}{\partial y}+\frac{\partial v}{\partial x}\right)^{2}} \quad \text { for both plane strain and stress states }
$$

Applying Eq. (14) and (16) to interface 1 and 2 shown in Fig.3a, and using the displacement jump's definition of Eq. (1), one gets the mechanical condition at modeled interface as

$$
\begin{gathered}
\sigma_{x A}=\frac{\frac{2}{\sqrt{3}(\sqrt{3} \beta)^{1 / m}}\left(\frac{\Delta u}{\delta}-\frac{\partial v}{\partial y}\right)}{\left[4\left(\frac{\Delta u}{\delta}-\frac{\partial v}{\partial y}\right)^{2}+\left(\frac{\partial u}{\partial y}+\frac{\Delta v}{\delta}\right)^{2}\right]^{\frac{m-1}{2 m}}}, \tau_{x y A}=\frac{\frac{1}{\sqrt{3}(\sqrt{3} \beta)^{1 / m}}\left(\frac{\partial u}{\partial y}+\frac{\Delta v}{\delta}\right)}{\left[4\left(\frac{\Delta u}{\delta}-\frac{\partial v}{\partial y}\right)^{2}+\left(\frac{\partial u}{\partial y}+\frac{\Delta v}{\delta}\right)^{2}\right]^{\frac{m-1}{2 m}}} \\
\sigma_{x B}=\frac{\frac{2}{\sqrt{3}(\sqrt{3} \beta)^{1 / m}}\left(\frac{\Delta u}{\delta}-\frac{\partial v}{\partial y}-\frac{\partial \Delta v}{\partial y}\right)}{\left[4\left(\frac{\Delta u}{\delta}-\frac{\partial v}{\partial y}-\frac{\partial \Delta v}{\partial y}\right)^{2}+\left(\frac{\partial u}{\partial y}+\frac{\partial \Delta u}{\partial y}+\frac{\Delta v}{\delta}\right)^{2}\right]^{\frac{m-1}{2 m}}} \\
\tau_{x y B}=\frac{1}{\sqrt{3}(\sqrt{3} \beta)^{1 / m}}\left(\frac{\partial u}{\partial y}+\frac{\partial \Delta u}{\partial y}+\frac{\Delta v}{\delta}\right) \\
{\left[4\left(\frac{\Delta u}{\delta}-\frac{\partial v}{\partial y}-\frac{\partial \Delta v}{\partial y}\right)^{2}+\left(\frac{\partial u}{\partial y}+\frac{\partial \Delta u}{\partial y}+\frac{\Delta v}{\delta}\right)^{2}\right]^{\frac{m-1}{2 m}}}
\end{gathered}
$$

where $u, v$ are displacements on modeled interface, and $\Delta u, \Delta v$ are displacement jumps. These relationships are the details of Eq. (2) for a non-linear (power law) interfacial layer. Strong interactions of displacement jumps on tractions can be understood from this relationship. Since all partial derivatives appeared in Eq. (17) can be expressed by $u, v, \Delta u, \Delta v$ at the modeled interface numerically, the bonding problem can be analyzed just with the use of Eq. (17), though non-linear analysis is necessary.

If contributions of partial derivatives of displacement can be neglected, Eq. (17) degrades to a nonlinear cohesive model as

$$
\sigma_{x A}=\sigma_{x B}=\frac{2 k_{n} \Delta u}{\left[4(\Delta u)^{2}+(\Delta v)^{2}\right]^{\frac{m-1}{2 m}}}, \quad \tau_{x y A}=\tau_{x y B}=\frac{k_{n} \Delta v}{\left[4(\Delta u)^{2}+(\Delta v)^{2}\right]^{\frac{m-1}{2 m}}}
$$

$$
k_{n}=\frac{1}{\sqrt{3}(\sqrt{3} \beta \delta)^{\frac{1}{m}}}
$$


Eq. (17) gives the mechanical condition of complete interface model, while Eq. (18) may be a valuable reference in assuming a non-linear cohesive model.

\subsection{Identifying method of interface property}

From Section 2, it can be seen that three parameters are required to describe the interface property. That is, equivalent shear or Young's modulus and Poisson's ratio of interfacial layer, together with its thickness $\delta$. The thickness might be in micro-scale, and can be measured by micro observations such as SEM or other methods. While for metal-like intermediate, the Poisson's ratio is near to 0.3 . The shear modulus or Young's modulus might be detected by Nano-indentation method. Of course, it could be determined by matching analysis results with measured displacement near interface.

\section{Conclusions}

Regarding the interfacial phase as an equivalent elastic intermediate, a complete interface model has been developed. The main results can be concluded as

1) Due to the physical interface phase, not only the displacement jump, but also the traction jump, are possible across the interface. The complete interface model can deal well with these jumps, and give the correct analysis results.

2) For a hard interfacial layer, classic interface model can obtain reasonable reactions on interface, but it is not valid for soft interfacial layer.

3) Cohesive model can also give reasonable traction results, but only if the spring constants are assumed properly. For a soft interfacial layer, the spring constants can be calculated from interfacial property. But for a hard layer, proper spring constants have to be determined by matching other results.

4) Stress component parallel to the interface can be analyzed by the complete interface model, which could be very large in soft interfacial layer.

\section{Acknowledgments:}

This work is supported by the National Natural Science Foundation of China (NSFC) under grant No.11772189.

\section{References}

1. Rizzoni, R., Dumont, S., Lebon, F., and Sacco, E. Higher order model for soft and hard elastic interfaces. Int. J. Solids and Struct., 2014, Volume 51(23), pp.4137-4148, https://doi.org/10.1016/j.ijsolstr.2014.08.005.

2. Lebon, F. and Rizzoni, R. Asymptotic analysis of a thin interface: The case involving similar rigidity. Int. J. Eng. Sci., 2010, 48(5), 473-486, https://doi.org/10.1016/j.ijengsci.2009.12.001.

3. Lebon, F., Khaoua, A. O., and Licht, C. Numerical study of soft adhesively bonded joints in finite elasticity. Comp. Mech., 1998, Volume 21(2), pp.134-140, https://doi.org/10.1007/s004660050289.

4. Dunders J., Boundary condition at interface, In Micromechanics and in homogeneous, Ed. Weng G.J., 1990, Springer Verlag, pp.109-114.

5. Suresh S. and Needleman A., Interfacial phenomena in composites, in: Elsevier applied science, Elsevier, New York, US., 1989, pp. 1-12.

6. Benveniste, Y. A general interface model for a three-dimensional curved thin anisotropic interphase between two anisotropic media. J. Mech. Phy. Solids, 2006, Volume 54(4), pp. 708-734, https://doi.org/10.1016/i.jmps.2005.10.009.

7. Xu J.Q. Mutoh Y. and Fu L.D., A Normal Force on the Free Surface of a Coated Material. J. Elasticity, 2003, Volume 73, pp.147-164, https://doi.org/10.1023/B:ELAS.0000029934.93885.11.

8. Bogy D.B., Edge bonded dissimilar orthogonal elastic wedges under normal and shear loadings, J.Appl.Mech., 1968, Volume 35, pp.146-154, http://dx.doi.org/10.1115/1.3601236.

9. Rice J.R., Elastic fracture mechanics concepts for interfacial cracks. J.Appl.Mech., 1988, Volume 55, pp.98103, http://dx.doi.org/10.1115/1.3173668.

10. Elices, M., Guinea, G. V., Gomez, J., and Planas, J. The cohesive zone model: advantages, limitations and challenges. Eng. Fract. Mech., 2002, Volume 69(2), 137-163, https://doi.org/10.1016/S0013-7944(01)00083-2. 
11. Valoroso, N., Sessa, S., Lepore, M., and Cricri, G. Identification of mode-I cohesive parameters for bonded interfaces based on DCB test. Eng. Fract. Mech., 2013, Volume 104, pp.56-79, https://doi.org/10.1016/j.engfracmech.2013.02.008.

12. Özdemir, I., Brekelmans, W. A. M., and Geers, M. G. D. A thermo-mechanical cohesive zone model. Comput. Mech., 2010, Volume 46(5), pp.735-745, https://doi.org/10.1007/s00466-010-0507-z.

13. Corigliano, A. Formulation, identification and use of interface models in the numerical analysis of composite delamination. Int. J. Solids Struct., 1993, Volume 30(20), pp.2779-2811, https://doi.org/10.1016/0020-7683(93)90154-Y.

14. Rabinovitch, O. An extended high order cohesive interface approach to the debonding analysis of FRP strengthened beams. Int. J. Mech. Sci., 2014, Volume 81, pp.1-16, https://doi.org/10.1016/j.ijmecsci.2014.01.013.

15. Alfano G. On the influence of the shape of the interface law on the application of cohesive-zone models. Comp. Sci.Tech., 2006, Volume 66(6), pp.723-730, https://doi.org/10.1016/j.compscitech.2004.12.024.

16. Cai X.J and Xu J.Q., Interface models for thin interfacial layers, Appl. Math. Mech. 2016, Volume 37(6), pp.707 - 724, https://doi.org/10.1007/s10483-016-2084-6.

17. Yuuki R. and Xu J.Q., Boundary element analysis of dissimilar materials and interface crack, Comput. Mech., 1994, Volume 14, pp.116-127, https://doi.org/10.1007/BF00350279.

18. Xu, J. Q., Fu, L. D., and Mutoh, Y. A method for determining elastic-plastic stress singularity at the interface edge of bonded power law hardening materials. JSME Int. J., 2002, Volume 45, pp.177-183, http://doi.org/10.1299/jsmea.45.177. 